\title{
RESTRICTED LIE ALGEBRAS AND SIMPLE ASSOCIATIVE ALGEBRAS OF CHARACTERISTIC $p$
}

\author{
BY \\ G. HOCHSCHILD
}

Introduction. Let $F$ be a field of characteristic $p$, and let $C$ be a finite algebraic extension field of $F$ such that $C^{p} C F$. It has been shown in [4] that there is a one to one correspondence (up to isomorphisms) between the simple finite dimensional algebras with center $F$ and containing $C$ as a maximal commutative subring and the regular restricted Lie algebra extensions of $C$ by the derivation algebra of $C / F$. This led to a very simple description of the group of similarity classes of these algebras. The main task of the present paper is to investigate the connection between restricted Lie algebras and simple associative algebras of characteristic $p$ quite generally. For this purpose, we generalize the notion of a regular extension of $C$ by the derivation algebra of $C / F$ to that of a regular extension of a simple algebra $A$ with center $C$ by the derivation algebra of $C / F$. The correspondence mentioned above is then generalized to a one to one correspondence between these extensions and the simple algebras with center $F$ which contain $A$ as the commutator algebra of $C$ (Theorem 3 ). We shall then show how every simple algebra containing a purely inseparable extension field of its center as a maximal commutative subring can be built up in a series of steps from regular Lie algebra extensions. With a suitable composition of regular extensions, which we define in $\$ 2$, this reduces the structure of the group of algebra classes with a fixed purely inseparable splitting field to the structural elements of restricted Lie algebras, at least in principle. However, it does not yield a direct description of this group, as was the case for splitting fields of exponent one.

In $\S 3$ we show that the tensor multiplication with a purely inseparable extension field $C$ of $F$ maps the Brauer group of algebra classes over $F$ onto the Brauer group over $C$ (Theorem 5). Although this result seems not to have been stated before, it may well be regarded as one of the culminating points of Albert's theory of $p$-algebras. In fact, Chapter VII of [1] contains all the essential points of a proof along classical lines, which is the first proof we give in $\S 3$. Here, the main tools are Galois theory and the theory of cyclic $p$-extensions. Our second proof is entirely different. It proceeds within the framework of the first part of this paper, replacing the field theory with the theory of Lie algebras. In particular, we use the theory of restricted Lie algebra kernels, [3], in order to show that if $A$ is a simple algebra with center $C$ and $C^{p} \subset F$ then there always exists a regular extension of $A$ by the derivation algebra of $C / F$, whence (by Theorem 3 ) $A$ can be imbedded as the

Received by the editors December 4, 1954. 
commutator algebra of $C$ in a simple algebra with center $F$. Theorem 5 then follows almost immediately.

1. Regular extensions. Let $F$ be a field of characteristic $p \neq 0$, and let $C$ be a finite algebraic extension of $F$ such that $C^{p} C F$. Let $T$ be the derivation algebra of $C / F$, regarded as a restricted Lie algebra over $F$. Observe that $T$ has also the structure of a vector space over $C$ in the natural way. The scalar multiplications are connected with the commutation in $T$ by the identity $\left[c_{1} t_{1}, c_{2} t_{2}\right]=c_{1} t_{1}\left(c_{2}\right) t_{2}-c_{2} t_{2}\left(c_{1}\right) t_{1}+c_{1} c_{2}\left[t_{1}, t_{2}\right]$, where $c_{i} \in C$ and $t_{i} \in T$. Furthermore, by Lemma 1 of [4], the scalar multiplications are connected with the $p$ th power map in $T$ by the identity $(c t)^{p}=c^{p} t^{p}+(c t)^{p-1}(c) t$.

Now let $A$ be a simple associative algebra with center $C$. Let $S$ be a restricted Lie algebra over $F$, and suppose that $A$, with its natural structure as a restricted Lie algebra over $F$, is contained as an ideal in $S$ in such a way that, for every $s \in S$, the restriction to $A$ of the inner derivation effected by $s$ is even a derivation of the associative algebra $A$, i.e., that for all $a_{i} \in A$, we have

$$
\left[s, a_{1} a_{2}\right]=\left[s, a_{1}\right] a_{2}+a_{1}\left[s, a_{2}\right] .
$$

Then these derivations map $C$ into itself, and if $\phi(s)$ denotes the restriction to $C$ of the inner derivation effected by $s, \phi$ is a restricted Lie algebra homomorphism of $S$ into $T$. We shall say that the pair $(S, \phi)$ constitutes a regular extension of $A$ by $T$ if the kernel of $\phi$ coincides with $A, \phi(S)=T$, and $S$ can be given the structure of a vector space over $C$, extending the $C$-space structure of $A$, such that $\phi$ is $C$-linear and the following regularity conditions hold, for all $c, c_{i}$ in $C$ and all $s, s_{i}$ in $S$ :

$$
\begin{aligned}
{\left[c_{1} s_{1}, c_{2} s_{2}\right] } & =c_{1} \phi\left(s_{1}\right)\left(c_{2}\right) s_{2}-c_{2} \phi\left(s_{2}\right)\left(c_{1}\right) s_{1}+c_{1} c_{2}\left[s_{1}, s_{2}\right] \\
(c s)^{p} & =c^{p} s^{p}+\phi(c s)^{p-1}(c) s .
\end{aligned}
$$

Such a $C$-space structure on $S$ will then be called an admissible $C$-space structure. We say that two such regular extensions of $A$ by $T$ are equivalent if there exists a restricted Lie algebra isomorphism $\alpha$ of $S$ onto $S^{\prime}$ which leaves the elements of $A$ fixed and for which $\phi^{\prime} \alpha=\phi$.

If $B$ is a simple finite dimensional algebra with center $F$ and containing $C$ then the commutator algebra $A$ of $C$ in $B$ is simple and has center $C$. Let $S$ be the set of all elements $s \in B$ for which $s c-c s \in C$ whenever $c \in C$, and let $\phi(s)$ denote this derivation $c \rightarrow s c-c s$ of $C$. Then it is easily seen (using Lemma 1 of $[4]$ in order to verify condition (ii)) that $(S, \phi)$ is a regular extension of $A$ by $T$.

In order to deal adequately with regular extensions we require the following auxiliary notion. A simple finite dimensional algebra $A$ with center $C$ may be regarded as a full matrix algebra $D_{n}$ where $D$ is division algebra with center $C$. There exists a separable extension field $Z$ of $C$ which is a maximal 
commutative subring of $D$. Let $R$ denote the subring of $A$ which consists of all those matrices whose diagonal entries lie in $Z$ and whose other entries are 0 . Then $R$ contains $C$ and is $C$-isomorphic with the direct sum of $n$ copies of $Z$. Thus $R$ is a separable commutative algebra over $C$. Furthermore, $R$ is a maximal commutative subring of $A$. Generally, if $R$ is any maximal commutative subring of $A$ which contains $C$ and is separable over $C$, we shall say that $R$ is a regulator of $A$. We have then $C R^{p}=R$, and if $F$ is a subfield of $C$ as above, $F R^{p}$ is separable over $F$.

Theorem 1. Let $F$ be a field of characteristic $p \neq 0, C$ a finite algebraic extension field of $F$ such that $C^{p} C F, T$ the derivation algebra of $C / F$. Let $A$ be a simple finite dimensional algebra with center $C$, and let $(S, \phi)$ be a regular extension of $A$ by $T$. Then, for any regulator $R$ of $A$ and any admissible $C$-space structure of $S$, there exists a $C$-linear Lie algebra isomorphism $\psi$ of $T$ into $S$ such that, for any $t \in T, \phi \psi(t)=t$, the inner derivation $D_{\psi(t)}$ effected by $\psi(t)$ maps $R$ into itself, and $\psi(t)^{p}-\psi\left(t^{p}\right) \in R^{p}$.

Proof. Let $S^{\prime}$ be the restricted subalgebra of $S$ which consists of all those elements $s \in S$ for which $D_{s}(R) \subset R$. Since $R$ is separable over $C$, every derivation $t$ of $C$ can be extended (uniquely) to a derivation $t^{\prime}$ of $R$. Let $s \in S$ and consider the restriction to $R$ of $D_{s}$; call this $\delta_{s}$. Then $\delta_{s}-\phi(s)^{\prime}$ is a derivation of $R$ into $A$ which annihilates $C$. Since $R$ is a semisimple subalgebra of $A$, we can apply a well known result of Jacobson's $\left({ }^{1}\right)$ to conclude that there is an element $a \in A$ such that $\delta_{s}-\phi(s)^{\prime}$ coincides with $\delta_{a}$. Now $\delta_{s-a}=\phi(s)^{\prime}$ and therefore maps $R$ into itself, which means that $s-a \in S^{\prime}$. Hence $\phi\left(S^{\prime}\right)=T$, and if $\phi^{\prime}$ denotes the restriction of $\phi$ to $S^{\prime}$ then $\left(S^{\prime}, \phi^{\prime}\right)$ is a restricted Lie algebra extension of $A \cap S^{\prime}$ by $T$. Since $R$ is commutative, every derivation of $R$ annihilates $R^{p}$. Hence every element of $A \cap S^{\prime}$ commutes with every element of $R^{p}$, and therefore also with every element of $C R^{p}=R$. Hence $A \cap S^{\prime}=R$, so that $\left(S^{\prime}, \phi^{\prime}\right)$ is a regular extension of $R$ by $T$. Now the proof of Theorem 4 of [4] applies without any changes to this extension $\left(S^{\prime}, \phi^{\prime}\right)$ and gives the desired result.

Furthermore, one can now proceed exactly as in that proof to obtain:

Theorem 2. Let $(S, \phi)$ and $\left(S^{\prime}, \phi^{\prime}\right)$ be any two equivalent regular extensions of $A$ by $T$. Then, for any given admissible $C$-space structures of $S$ and $S^{\prime}$, there is a $C$-linear equivalence isomorphism of $S$ onto $S^{\prime}$.

Let $(S, \phi)$ be a regular extension of $A$ by $T$, and let $U_{S}$ denote the restricted universal enveloping algebra of the restricted Lie algebra $S\left({ }^{2}\right)$. Let $U_{S}^{+}$denote the ideal of $U_{S}$ which is generated by the canonical images $s^{\prime}$ in $U_{S}$ of the elements $s \in S$. Now equip $S$ with an admissible $C$-space structure,

(1) See Theorem 7 of [5], or Theorem 3 of [4].

(2) For this notation, and for the results we take from the theory of restricted Lie algebras (due to Jacobson) and their cohomology groups, see [2]. 
and let $J$ be the ideal of $U_{S}^{+}$which is generated by the elements of the form $c^{\prime} s^{\prime}-(c s)^{\prime}$, with $c \in C$ and $s \in S$. Put $V_{S}=U_{S}^{+} / J$. Then $V_{S}$ is a finite dimensional associative algebra over $F$. It is clear from Theorem 2 that, although the construction of $V_{S}$ involves the choice of an admissible $C$-space structure on $S, V_{S}$ is determined up to an isomorphism by the equivalence class of the extension $(S, \phi)$. More precisely, if $\alpha$ is a $C$-linear equivalence isomorphism of $(S, \phi)$ onto $\left(S^{\prime}, \phi^{\prime}\right)$, then $\alpha$ induces an isomorphism $\alpha^{*}$ of $V_{S}$ onto $V_{S^{\prime}}$, such that if $\rho$ and $\rho^{\prime}$ are the natural maps of $S$ into $V_{S}$ and of $S^{\prime}$ into $V_{S^{\prime}}$, respectively, we have $\alpha^{*} \rho=\rho^{\prime}$. We are now in a position to prove the following generalization of Theorem 5 of [4].

TheOREM 3. Let $(S, \phi)$ be a regular extension of $A$ by $T$. Then the canonical map of $S$ into $V_{S}$ is $1-1$, and its restriction to $A$ is an associative algebra isomorphism by means of which we identify $A$ with a subalgebra of $V_{S} . V_{S}$ is then a finite dimensional simple algebra with center $F$, and $A$ is the commutator algebra of $C$ in $V_{S}$. Furthermore, the canonical image of $S$ in $V_{S}$ coincides with the set of all elements $v \in V_{S}$ for which $D_{v}(C) \subset C$, and the corresponding regular extension of $A$ by $T$ is equivalent with $(S, \phi)$ by the canonical map of $S$ into $V_{S}$. Conversely, if $B$ is a finite dimensional simple algebra with center $F$ and containing $C$, if $A$ is the commutator algebra of $C$ in $B$, and if $(S, \phi)$ is the resulting regular extension of $A$ by $T$, then the homomorphism of $U_{S}^{+}$into $B$ which extends the injection $S \rightarrow B$ induces an isomorphism of $V_{S}$ onto $B$.

Proof. We have $C=F\left[u_{1}, \cdots, u_{k}\right]$ and corresponding derivations $t_{1}, \cdots, t_{k}$ of $C$ such that $t_{i}\left(u_{j}\right)=\delta_{i j} u_{j}$, whence $t_{i} t_{j}=t_{j} t_{i}$ and $t_{i}^{p}=t_{i}$. (Cf. $\S 1$ of [4]). Let $R$ be a regulator of $A$, and let $\psi$ be a $C$-linear Lie algebra isomorphism of $T$ into $S$ such as is given by Theorem 1 . Write $\psi\left(t_{i}\right)=s_{i}$. Then $s_{i}^{p}=s_{i}+r_{i}$, with $r_{i} \in R^{p}$. Put $F R^{p}=R_{0}$, and construct the commutative polynomial ring $R_{0}\left[x_{1}, \cdots, x_{k}\right]$ in elements $x_{i}$ which are algebraically independent over $R_{0}$. Let $I$ be the ideal of this polynomial ring which is generated by the elements $x_{i}^{p}-x_{i}-r_{i}$. Then $R_{0}\left[x_{1}, \cdots, x_{k}\right] / I=R_{0}\left[y_{1}, \cdots, y_{k}\right]$, where $y_{i}$ is the coset of $x_{i}$, so that $y_{i}^{p}=y_{i}+r_{i}$. Put $V=A \otimes_{R_{0}} R_{0}\left[y_{1}, \cdots, y_{k}\right]$, this tensor product being taken for the natural structure of $A$ as a right $R_{0}$-module and the natural structure of $R_{0}\left[y_{1}, \cdots, y_{k}\right]$ as a left $R_{0}$-module. We regard $V$ as a left $A$-module in the natural fashion. Since the inner derivation effected by $s_{i}$ maps $R$ into itself, it must annihilate $R^{p}$ and hence $R_{0}$. It follows that there is a unique additive endomorphism $z_{i}$ of $V$ such that, for $a \in A$ and $y \in R_{0}\left[y_{1}, \cdots, y_{k}\right], z_{i}(a \otimes y)=\left[s_{i}, a\right] \otimes y+a \otimes y_{i} y$. We may identify the elements of $A$ with the module operations they effect in $V$, and it is easy to check that we have then $z_{i} a-a z_{i}=\left[s_{i}, a\right]$, and $z_{i}^{p}=z_{i}+r_{i}$. Also, we evidently have $z_{i} z_{j}=z_{j} z_{i}$. Let $M$ be the ring of endomorphisms of $V$ which is generated by the $z_{i}$ and the elements of $A$. Then it is easily seen that the monomials $z_{1}^{e_{1}} \cdots z_{k}^{e_{k}}$, with $0 \leqq e_{i}<p$, are (left) $A$-independent in $M$ and span $M$ over $A$.

Now consider an element of $M$, writing it in the form $m=m_{0}+m_{1} z_{i}+\ldots$ 
$+m_{q} z_{i}^{q}$, where $q<p$ and the $m_{r}$ are $A$-linear combinations of monomials in the $z_{j}$ with $j \neq i$. Then the $m_{r}$ commute with $u_{i}$, and it follows that $m u_{i}-u_{i} m$ $=q m_{q} u_{i} z_{i}^{q-1}+m^{\prime}$, where $m^{\prime}$ is of degree less than $q-1$ in $z_{i}$. It is easily seen from this that if $m$ commutes with every element of $C$ then $m \in A$. Hence the commutator algebra of $C$ in $M$ coincides with $A$. At the same time we see that the center of $M$ is contained in $A$, and hence also in the center $C$ of $A$. Since a central element commutes with every $z_{i}$, and since an element of $C$ which is annihilated by each $t_{i}$ must belong to $F$, it follows that the center of $M$ coincides with $F$. Finally, by repeatedly forming $m u_{i}-u_{i} m$ (with suitable choices of the index $i$ at each stage), starting with any nonzero element $m$, we eventually find a nonzero element of $A$ which belongs to the ideal $M m M$. Since $A$ is simple, we conclude then that $1 \in M m M$, whence $M m M$ $=M$. Thus $M$ is simple.

Now every element of $S$ can be written uniquely in the form

$$
s=a+\sum_{i=1}^{k} c_{i} s_{i}
$$

where $a \in A$ and $c_{i} \in C$. We define a $C$-linear map $\alpha$ of $S$ into $M$ by setting $\alpha(s)=a+\sum_{i=1}^{k} c_{i} z_{i}$. If we regard $M$ as a restricted Lie algebra over $F$ in the usual way, we see from the regularity conditions $\left({ }^{*}\right)$ and (i) that $\alpha$ is a Lie algebra isomorphism, and from the regularity condition (ii) that $\alpha$ is even a restricted Lie algebra isomorphism. Hence $\alpha$ can be extended uniquely to a homomorphism of $U_{S}^{+}$into $M$ which is evidently onto and annihilates the ideal $J$ by means of which we have defined $V_{S}$. Hence the canonical map of $S$ into $V_{S}$, which is a right factor of $\alpha$, must be 1-1. Clearly, the ordered monomials in the canonical images of the $s_{i}$ in $V_{S}$, with non-negative exponents less than $p$, span $V_{S}$ over the canonical image of $A$ in $V_{S}$. Hence we have the dimensionality relations $\left[V_{S}: F\right] \leqq p^{k}[A: F]=[M: F]$. It follows that the homomorphism of $V_{S}$ onto $M$ which is induced by $\alpha$ is actually an isomorphism. Hence we may identify $A$ with its canonical image in $V_{S}$, and $V_{S}$ is a simple algebra with center $F$ which contains $A$ as the commutator algebra of $C$. Furthermore, if $m$ is an element of $M$ such that $m c-c m \in C$, for every $c \in C$, our above computation of $m u_{i}-u_{i} m$ shows that $m \in \alpha(S)$. Hence $S$ may be identified with the set of all $v \in V_{S}$ for which $D_{v}(C) \subset C$, and then the corresponding regular extension of $A$ by $T$ evidently coincides with $(S, \phi)$.

There remains only to prove the last part of Theorem 3 . It is evident that the canonical homomorphism of $U_{S}^{+}$into $B$ which extends the injection $S \rightarrow B$ annihilates $J$ and therefore induces a homomorphism of $V_{S}$ into $B$ which evidently leaves the elements of $A$ fixed. Since we know already that $V_{S}$ is simple, we may conclude that this homomorphism is an isomorphism of $V_{S}$ into $B$. Also, we know that $\left[V_{S}: F\right]=p^{k}[A: F]=[C: F][A: F]$, and since $A$ is the commutator algebra of $C$ in $B$ we have $[C: F][A: F]=[B: F]$. Hence $\left[V_{S}: F\right]=[B: F]$, whence our isomorphism must map $V_{S}$ onto $B$. This com- 
pletes the proof of Theorem 3 .

Now let $A$ be a simple algebra with center $F$ which contains a purely inseparable extension field $C$ of $F$ as a maximal commutative subring. We can insert a sequence of fields $F=C^{(e)} \subset C^{(e-1)} \subset \cdots \subset C^{(0)}=C$ such that $F\left(C^{(r-1)}\right)^{p}=C^{(r)}$, for each $r$. Let $A^{(r)}$ be the commutator algebra of $C^{(r)}$ in $A$, and let $S^{(r)}$ be the set of all elements $a \in A$ for which $D_{a}\left(C^{(r)}\right) \subset C^{(r)}$. If $T^{(r)}$ denotes the derivation algebra of $C^{(r)} / C^{(r+1)}$, we have in the natural way the regular extensions $\left(S^{(r)}, \phi^{(r)}\right)$ of $A^{(r)}$ by $T^{(r)}$. It follows at once from Theorem 3 that $V_{S}{ }^{(r)}$ is isomorphic with $A^{(r+1)}$ by the map which extends the injection of $S^{(r)}$ into $A^{(r+1)}$. In particular, we conclude that the subalgebra of $A$ which is generated by the elements of $S^{(r)}$ coincides with $A^{(r+1)}$. Moreover, it is now clear how $A$ can be built up from $C$ by a sequence of regular extensions. If $C^{p} C F$ we have $e=1$, and we know from [4] that our construction establishes an isomorphism between the group of similarity classes of the simple algebras split by $C$ and the group of equivalence classes of the regular extensions of $C$ by $T$. It is therefore of interest to generalize the composition of regular extensions of $C$ by $T$ to a composition of our present more general regular extensions. This is the purpose of the next section.

2. Composites of regular extensions. Suppose we are given two regular extensions $(S, \phi)$ and $\left(S^{\prime}, \phi^{\prime}\right)$ of simple algebras $A$ and $A^{\prime}$, with the common center $C$, by the same derivation algebra $T$ of $C / F$. We shall define a composite regular extension of $A \otimes_{C} A^{\prime}$ by $T$. Let $D$ be the restricted subalgebra of the direct sum $\left(S, S^{\prime}\right)$ of $S$ and $S^{\prime}$ which consists of all elements $\left(s, s^{\prime}\right)$ in which $\phi(s)=\phi^{\prime}\left(s^{\prime}\right)$. Corresponding to each element $d=\left(s, s^{\prime}\right)$ of $D$, there is a unique derivation $\delta_{d}$ of $A \otimes_{c} A^{\prime}$ such that $\delta_{d}\left(a \otimes a^{\prime}\right)=[s, a] \otimes a^{\prime}+a \otimes\left[s^{\prime}, a^{\prime}\right]$. The map $d \rightarrow \delta_{d}$ is clearly a restricted Lie algebra homomorphism of $D$ into the derivation algebra of $A \otimes_{C} A^{\prime}$, relative to $F$. Now we regard $A \otimes_{C} A^{\prime}$ as a restricted Lie algebra over $F$ and use $\delta$ for defining the semidirect sum $U=\left(A \otimes_{C} A^{\prime}, D\right)_{8}$. The commutation in $U$ is given by the formula

$$
\left[\left(x_{1}, d_{1}\right),\left(x_{2}, d_{2}\right)\right]=\left(x_{1} x_{2}-x_{2} x_{1}+\delta_{d_{1}}\left(x_{2}\right)-\delta_{d_{2}}\left(x_{1}\right),\left[d_{1}, d_{2}\right]\right),
$$

where $x_{i} \in A \otimes_{C} A^{\prime}$ and $d_{i} \in D$. It is known from the elementary theory of restricted Lie algebras that there is one and only one $p$-map in $U$ which coincides with the given $p$-maps on the ideal $A \otimes_{c} A^{\prime}$ and on the complementary subalgebra $D$. When equipped with this $p$-map, $U$ is a restricted Lie algebra containing $A \otimes_{C} A^{\prime}$ as a restricted ideal in such a way that the regularity condition $\left(^{*}\right)$ is satisfied. Now let $V$ be the subspace of $U$ which is spanned by the elements $(a \otimes 1,-(a, 0))$, with $a \in A$, and the elements $\left(1 \otimes a^{\prime},-\left(0, a^{\prime}\right)\right)$, with $a^{\prime} \in A^{\prime}$. It is easily verified that $V$ is a restricted ideal in $U$. We put $E=U / V$. The canonical map of $A \otimes_{C} A^{\prime}$ into $E$ is evidently an isomorphism by means of which we shall identify $A \otimes_{C} A^{\prime}$ with a restricted ideal of $E$. The homomorphism $\phi$ (or $\phi^{\prime}$ ) induces a restricted homomorphism $\psi$ of $E$ onto $T$ whose kernel clearly coincides with $A \otimes_{C} A^{\prime}$. Hence $(E, \psi)$ is a 
restricted extension of $A \otimes_{C} A^{\prime}$ by $T$ which evidently satisfies the regularity condition $\left(^{*}\right)$. Furthermore, any admissible $C$-space structures on $S$ and $S^{\prime}$ induce a $C$-space structure on $U$, and hence on $E$, which evidently satisfies the regularity condition (i). In order to see that the regularity condition (ii) is also satisfied, we must make a precise analysis of the $p$-map. We must show that $(c e)^{p}=c^{p} e^{p}+D_{c s}^{p-1}(c) e$, for all $c \in C$ and all $e \in E$. This holds clearly if $e \in A \otimes_{C} A^{\prime}$, and also if $e$ is the canonical image in $E$ of an element of $D$. Hence it suffices to prove that if the formula holds for $e=e_{1}$ and for $e=e_{2}$ then it holds also for $e=e_{1}+e_{2}$. In order to do this, we consider the ordinary universal enveloping algebra $R_{E}$ of $E$. Let $I$ be the ideal of $R_{E}$ which is generated by the elements $c^{\prime} e^{\prime}-(c e)^{\prime}$, for $e \in E$ and $c \in C$, where $e \rightarrow e^{\prime}$ denotes the canonical isomorphism of $E$ into $R_{E}$. If $e_{1}$ is any other element of $E$, we have

$$
\begin{aligned}
e_{1}^{\prime}\left(c^{\prime} e^{\prime}-(c e)^{\prime}\right)-\left(c^{\prime} e^{\prime}-(c e)^{\prime}\right) e_{1}^{\prime} & =e_{1}^{\prime} c^{\prime} e^{\prime}-c^{\prime} e^{\prime} e_{1}^{\prime}+\left[c e, e_{1}\right]^{\prime} \\
& =c^{\prime}\left[e_{1}, e\right]^{\prime}-\left(c\left[e_{1}, e\right]\right)^{\prime}+\left[e_{1}, c\right]^{\prime} e^{\prime}-\left(\left[e_{1}, c\right] e\right)^{\prime} .
\end{aligned}
$$

Hence $I$ actually coincides with the right ideal which is generated by the elements $c^{\prime} e^{\prime}-(c e)^{\prime}$. Hence we see easily that $I \cap E^{\prime}=(0)$, so that we may identify the $C$-space $E$ with a subspace of $R_{E} / I$. Now let us momentarily indicate the $p$-map in $E$ by $e \rightarrow e^{[p]}$, and let $e^{p}$ stand for the $p$ th power of $e$ as an element of $R_{E} / I$, i.e., $e^{p}$ now denotes the coset $\bmod I$ of $\left(e^{\prime}\right)^{p}$. Observe that, by our definition of $I$, we have $c^{[p]}=c^{p}$, for every $c \in C$. It follows at once from the definition of a $p$-map in a restricted Lie algebra that the map $e \rightarrow e^{p}-e^{[p]}$ of $E$ into $R_{E} / I$ is additive. If we apply Lemma 1 of [4] in $R_{E} / I$ we find that $(c e)^{p}=c^{p} e^{p}+D_{c e}^{p-1}(c) e$, for all $c \in C$ and all $e \in E$. Now suppose that $e_{1}$ and $e_{2}$ are elements of $E$ which satisfy our regularity condition (ii), i.e., in our present notation, $\left(c e_{i}\right)^{[p]}=c^{p} e_{i}^{[p]}+D_{c e_{i}}^{p-1}(c) e_{i}$. In $R_{E} / I$, we have

$$
\left(c\left(e_{1}+e_{2}\right)\right)^{[p]}=\left(c\left(e_{1}+e_{2}\right)\right)^{p}-\left(c e_{1}\right)^{p}-\left(c e_{2}\right)^{p}+\left(c e_{1}\right)^{[p]}+\left(c e_{2}\right)^{[p]} .
$$

If we expand the first three terms on the right by using the regularity of $R_{E} / I$ as noted above, and the last two terms by using our hypothesis that (ii) holds for $e_{1}$ and $e_{2}$, we find, on recombining the terms, that (ii) holds for $e_{1}+e_{2}$. Hence $(E, \psi)$ is a regular extension of $A \otimes_{C} A^{\prime}$ by $T$.

THEOREM 4. Let $(E, \psi)$ be the composite of two regular extensions $(S, \phi)$ and $\left(S^{\prime}, \phi^{\prime}\right)$. Then $V_{E}$ is similar to $V_{S} \otimes_{F} V_{S^{\prime}}$

Proof. Let $V_{S}^{*}$ be the algebra of the right multiplications in $V_{S}$, and regard $V_{S}$ as a module for $V_{S} \otimes_{F} V_{S}^{*}$ in the natural fashion. Since $V_{S}$ is simple with center $F, V_{S} \otimes_{F} V_{S}^{*}$ is thereby faithfully represented as the algebra of all $F$-linear transformations of $V_{S}$. Now let $A$ be the kernel of $\phi$, so that $A$ is the commutator algebra of its center $C$ in $V_{S}$. Then our representation of $V_{S} \otimes_{F} V_{S}^{*}$ maps $A \otimes_{F} V_{S}^{*}$ isomorphically onto the algebra of all $C$-linear trans- 
formations of $V_{S}$, where we regard $V_{S}$ as a vector space over $C$, with $C$ operating by left multiplication on $V_{S}$. Similarly, we represent $A^{\prime} \otimes_{F} V_{\mathcal{S}^{\prime}}^{*}$ as the algebra of all $C$-linear transformations of $V_{S^{\prime}}$. Now let $W$ be the tensor product, relative to $C$, of the $C$-spaces $V_{S}$ and $V_{S^{\prime}}$; thus, $W$ is obtained from $V_{S} \otimes_{F} V_{S^{\prime}}$ by reducing mod the subspace spanned by the elements. $c v \otimes v^{\prime}$ $-v \otimes c v^{\prime}$, with $c \in C, v \in V_{S}$, and $v^{\prime} \in V_{S^{\prime}}$. The corresponding tensor product of our representations of $A \otimes_{F} V_{S}^{*}$ and $A^{\prime} \otimes_{F} V_{S}^{*}$, defines the structure of a module for $\left(A \otimes_{F} V_{S}^{*}\right) \otimes_{C}\left(A^{\prime} \otimes_{F} V_{S^{\prime}}^{*}\right)$ on $W$, and represents this algebra faithfully as the algebra of all $C$-linear transformations of $W$. We can write our tensor product in the form $\left(A \otimes_{C} A^{\prime}\right) \otimes_{F}\left(V_{S}^{*} \otimes_{F} V_{S^{\prime}}^{*}\right)$ and accordingly regard $W$ as a module for $V_{S}^{*} \otimes_{F} V_{S^{\prime}}^{*}$. This gives an imbedding of $A \otimes_{C} A^{\prime}$ in the algebra $B$ of all $V_{S}^{*} \otimes_{F} V_{S^{\prime}}^{*}$ endomorphisms of $W$, whereby $A \otimes_{C} A^{\prime}$ is identified with the commutator algebra of $C$ in $B$. By a standard result of the theory of representations of simple algebras, $B$ is similar to $V_{S} \otimes_{F} V_{S^{\prime}}$. Hence it will suffice to prove that $V_{E}$ is isomorphic with $B$. In doing this, we shall use the notation of the definition of $(E, \psi)$. An element $\left(s, s^{\prime}\right)$ of $D$ determines a unique element of $B$ which sends an element $v \otimes v^{\prime}$ of $W$ into $s v \otimes v^{\prime}+v \otimes s^{\prime} v^{\prime}$. If this map of $D$ into $B$ is augmented by the canonical injection of $A \otimes_{C} A^{\prime}$ into $B$, there results a restricted Lie algebra homomorphism of $U$ into $B$ whose kernel is easily seen to coincide with $V$. Hence we obtain a restricted Lie algebra isomorphism $\beta$ of $E$ into $B$ which leaves the elements of $A \otimes_{C} A^{\prime}$ fixed. Since $A \otimes_{C} A^{\prime}$ is the commutator algebra of $C$ in $B$, the regular extension corresponding to the pair $C \subset B$ is a regular extension $(R, \rho)$ of $A \otimes_{C} A^{\prime}$ by $T$, and we have evidently $\beta(E) \subset R$ and $\rho \beta=\psi$. This is enough to conclude that $\beta$ is an equivalence isomorphism of $(E, \psi)$ onto $(R, \rho)$. Hence $V_{E}$ is isomorphic with $V_{R}$, which is isomorphic with $B$, by Theorem 3. This completes the proof of Theorem 4.

Now let $B_{F}$ denote the group of similarity classes of the simple algebras with center $F$, and similarly define $B_{C}$, where $C$ is an extension field of $F$ with $C^{p} C F$. The map $A \rightarrow A \otimes_{F} C$ induces a homomorphism $\gamma$ of $B_{F}$ into $B_{C}$ whose kernel is the group $B_{F}(C)$ of the similarity classes of those simple algebras with center $F$ which are split by $C$. We shall show in the next section that $\gamma\left(B_{F}\right)=B_{C}$. For the moment, we merely recall the well known fact that, quite generally, the similarity class of an algebra $A$ with center $C$ belongs to $\gamma\left(B_{F}\right)$ if and only if there exists a simple algebra $U$ with center $F$ such that $A$ is contained as the commutator algebra of $C$ in $U$. In fact, suppose first that such an algebra $U$ exists. We may regard $U$ as a module for $C \otimes_{F} U^{*}$ in the natural way. Then we see that $A$ may be identified with the algebra of all $C \otimes_{F} U^{*}$. endomorphisms of $U$, whence it follows that $A$ is similar to $U \otimes_{F} C$. Conversely, let us suppose that there is a simple algebra $V$ with center $F$ such that $A$ is similar to $A^{\prime}=V \otimes_{F} C$. Put $[C: F]=n$. Then we can imbed $C$ in the full matrix algebra $F_{n}$ over $F$ so that it coincides with its own commutator algebra in $F_{n}$. Hence we can identify $A^{\prime}$ with the commutator algebra of $C$ 
in $V \otimes_{F} F_{n}$. Since $A$ is similar to $A^{\prime}$, there are positive integers $m$ and $m^{\prime}$ such that the full matrix algebra $A_{m}$ over $A$ is isomorphic with $A_{m^{\prime}}^{\prime}$. Since $A^{\prime}$ is the commutator algebra of $C$ in $V \otimes_{F} F_{n}=V_{n}, A_{m^{\prime}}^{\prime}$ may be identified with the commutator algebra of $C$ in $\left(V_{n}\right)_{m^{\prime}}=V_{n m^{\prime}}$. Since $A_{m^{\prime}}^{\prime}$ is isomorphic with $A_{m}$, it contains a copy of $F_{m}$, and therefore $V_{n m^{\prime}}$ contains a copy of $F_{m}$. Hence we may write $V_{n m^{\prime}}=U \otimes_{F} F_{m}$, where $U$ is the commutator algebra of this copy of $F_{m}$ in $V_{n m^{\prime}}$. Since $A$ is the commutator algebra of $F_{m}$ in $A_{m}$, our isomorphism of $A_{m}$ into $V_{n m^{\prime}}$ (via $A_{m^{\prime}}^{\prime}$ ) must map $A$ into $U$. Finally, since $A$ is the commutator algebra of $C_{m}$ in $A_{m}$, the image of $A$ in $U \otimes_{F} F_{m}$ is the commutator algebra of $C \otimes_{F} F_{m}$ in the image of $A_{m}$, i.e., in the commutator algebra of $C$ in $U \otimes_{F} F_{m}$ which, in any case, must contain the commutator algebra of $C \otimes_{F} F_{m}$. Hence the image of $A$ is the commutator algebra of $C \otimes_{F} F_{m}$ in $U \otimes_{F} F_{m}$, i.e., the commutator algebra of $C$ in $U$. This completes the proof of the characterization of $\gamma\left(B_{F}\right)$, in the general case.

Now let $A$ be a simple algebra with center $C$, and suppose that the similarity class of $A$ belongs to $\gamma\left(B_{F}\right)$. By the above, every similarity class in $B_{F}$ which maps into the similarity class of $A$ has a unique (to within isomorphisms) representative $U$ such that $A$ is contained in $U$ as the commutator algebra of $C$. Hence, by Theorem 3 , the preimage in $B_{F}$ of the similarity class of $A$ is in one to one correspondence with the set of equivalence classes of the regular extensions of $A$ by $T$. The coset structure of this preimage, with respect to the subgroup $B_{F}(C)$, is reflected in the set of equivalence classes of the regular extensions of $A$ by $T$ by their composition with the regular extension classes of $C$ by $T$, as follows at once from Theorem 4 . Furthermore, if $A^{\prime}$ is a second simple algebra with center $C$, then the preimage in $B_{F}$ of the similarity class of $A \otimes_{C} A^{\prime}$ is represented by the composites of the regular extensions of $A$ by $T$ with the regular extensions of $A^{\prime}$ by $T$.

Next we observe that an algebra $A$ with center $C$ is of the form $U \otimes_{F} C$, where $U$ is a simple algebra with center $F$ if and only if there exists a trivial regular extension of $A$ by $T$. Indeed, it is evident that $U \otimes_{F} C$ has a trivial extension by $T$. Conversely, suppose that $(S, \phi)$ is a trivial extension of $A$ by $T$. This means that there exists a restricted Lie algebra isomorphism $\psi$ of $T$ into $S$ such that $\phi \psi$ is the identity map of $T$ onto itself. Then $\psi(T)+C$ is a restricted subalgebra of $S$ which, together with the restriction of $\phi$ to $\psi(T)$ $+C$, constitutes a trivial regular extension of $C$ by $T$. The corresponding simple algebra with center $F$ is evidently a subalgebra of $V_{S}$. On the other hand, we know from [4] that it is the full matrix algebra $F_{n}$, where $n=[C: F]$. Hence we may write $V_{S}=U \otimes_{F} F_{n}$, where $U$ is the commutator algebra of $F_{n}$ in $V_{s}$. Now $C$ is contained in $F_{n}$ and coincides with its commutator algebra in $F_{n}$, while $A$ is the commutator algebra of $C$ in $V_{S}$. Hence $A=U \otimes_{P} C$, and clearly $U$ is simple with center $F$.

3. Existence of regular extensions and related questions. It is clear from the above that the following result implies the existence of regular extensions. 
TheOREM 5. Let $F$ be a field of characteristic $p$, and let $C$ be a finite algebraic extension of $F$ which is purely inseparable over $F$. Then every finite dimensional simple algebra $A$ with center $C$ is similar to a tensor product $B \otimes_{F} C$, where $B$ is a simple algebra with center $F$.

Proof. The algebra $A$ is similar to a tensor product $U \otimes_{C} V$, where the exponent of $U$ (in the group of algebra classes over $C$ ) is not divisible by $p$, while the exponent of $V$ is a power $p^{n}$ of $p$. Furthermore, we may take $U$ to be a crossed product, and it suffices to prove Theorem 5 in the two cases $A=U$, or $A=V$. The case $A=U$ is quite easy. We write $U=Z(G, f)$, where $Z / C$ is a normal extension with Galois group $G$, and $f$ is a factor set for $G$ in $Z$. Let $u$ denote the exponent of $U$. Then $f^{u}$ is a trivial factor set. Let $e$ be the exponent of $C$ relative to $F$, so that $C^{p^{*}} C F$. Since $p$ does not divide $u$, we can find integers $a$ and $b$ such that $a u+b p^{e}=1$. Then the factor set $f^{b p^{e}}$ is equivalent to $f$ and takes its values in $Z^{p^{\circ}}$. Since $C^{p^{\bullet}} C F$, and $Z$ is separable over $C$, $Z$ is isomorphic with $F Z^{p^{e}} \otimes_{F} C$. Furthermore, $F Z^{p^{a}}$ is normal over $F$, and the restriction map sends $G$ isomorphically onto the Galois group of $F Z^{p^{\circ}} / F$, which we may therefore identify with $G$. But then we can form the crossed product $F Z^{p^{\circ}}\left(G, f^{b p^{\theta}}\right)=U^{\prime}$, say; and clearly $U$ is isomorphic with $U^{\prime} \otimes_{F} C$. Now consider the more difficult case $A=V$. Since $V$ has exponent $p^{n}, V$ has a cyclic normal splitting field of degree a power of $p$. This is shown in Chap. VII of [1]. Actually, it suffices to use the easier result that $V$ is similar to a tensor product of cyclic crossed products of degree a power of $p$. In any case, it suffices to dispose of the case where $V$ is a crossed product of a cyclic extension field $Z$ of $C$, with $[Z: C]=p^{t}$. The case $t=0$ being trivial, we have to dispose of the case $t>0$. But then it is known from Albert's theorem on cyclic extensions of $p$-power degree of a field of characteristic $p$ that there exists a cyclic extension $Y / C$ such that $Z \subset Y$ and $[Y: C]=p^{i+e}$. Let $\sigma$ be a generator for the Galois group of $Y / C$, and let $\sigma^{\prime}$ be the restriction of $\sigma$ to $Z$. Then $\sigma^{\prime}$ is a generator for the Galois group of $Z / C$, and we have $V=Z\left(\sigma^{\prime}, c\right)$, with $c \in C$. Now $Z \subset Y$ and $[Y: Z]=p^{\circ}$. Hence, by a well-known result on cyclic algebras, $V$ is similar to the crossed product $Y\left(\sigma, c^{p^{0}}\right)$. (See Lemma 11, Chap. VII of [1]; this result is due to Albert, and Hasse). Since $c^{p^{*}} \in F$, we can now make the same argument as above (for $U$ ) to conclude that $V$ is similar to $F Y^{p^{e}}\left(\sigma, c^{p^{\bullet}}\right) \otimes_{F} C$. This completes the proof of Theorem 5 .

Theorem 5 implies that every derivation of $C / F$ can be extended to a derivation of $A$. Actually, a more general result on derivations holds over arbitrary fields (also in characteristic 0 ) and can be proved in a completely elementary fashion. Our result extends Jacobson's result, which we have already used in the proof of Theorem 1, that if $A$ is a finite dimensional simple algebra with center $C$, and if $B$ is a semisimple subalgebra of $A$ containing $C$, then every derivation of $B$ into $A$ which annihilates $C$ can be extended to an inner derivation of $A$. 
THEOREM $6^{(3)}$. Let $A$ be a simple algebra of finite dimension over its center $C$, and let $B$ be a semisimple subalgebra of $A$ containing $C$. Then every derivation of $B$ into $A$ which maps $C$ into itself can be extended to a derivation of $A$.

Proof. We observe first that it will suffice to prove that every derivation of $C$ can be extended to a derivation of $A$. Indeed, let $t$ be a derivation of $B$ into $A$ which maps $C$ into itself, and let $t_{C}$ be the restriction of $t$ to $C$. Suppose that $t_{C}$ can be extended to a derivation $s$ of $A$, and let $s_{B}$ be the restriction of $s$ to $B$. Then $t-s_{B}$ is a derivation of $B$ into $A$ which annihilates $C$, and hence, by the result of Jacobson's cited above, can be extended to a derivation $u$ of $A$. Now $s+u$ is a derivation of $A$ whose restriction to $B$ coincides with $t$.

The extendability of a derivation of $C$ to a derivation of $A$ depends only on the similarity class of $A$. For, suppose that a given derivation $t$ of $C$ can be extended to a derivation $t^{\prime}$ of a simple algebra $A^{\prime}$ which is similar to $A$. There are positive integers $m$ and $m^{\prime}$ such that $A_{m}$ is isomorphic with $A_{m^{\prime}}^{\prime}$. Let $t_{m^{\prime}}^{\prime}$ be the map of $A_{m^{\prime}}^{\prime}$ into itself which sends the matrix with entries $a_{i j}^{\prime}$ into the matrix with entries $t^{\prime}\left(a_{i j}^{\prime}\right)$. Then $t_{m^{\prime}}^{\prime}$ is evidently a derivation of $A_{m^{\prime}}^{\prime}$ which extends $t$. The isomorphism between $A_{m}$ and $A_{m^{\prime}}^{\prime}$ transports $t_{m^{\prime}}^{\prime}$ into a derivation $s$ of $A_{m}$ which extends $t$. On the other hand, $t$ can be extended to the derivation $t_{m}$ of $C_{m}$ (defined in the same way as $t_{m^{\prime}}^{\prime}$ was defined above). Let $s_{C_{m}}$ be the restriction of $s$ to $C_{m} \subset A_{m}$. Then $s_{C_{m}}-t_{m}$ is a derivation of $C_{m}$ into $A_{m}$ which annihilates $C$, and can therefore be extended to a derivation $u$ of $A_{m}$. Now consider the derivation $s-u$ of $A_{m}$. Its restriction to $C_{m}$ coincides with $t_{m}$, whence $s-u$ maps $C_{m}$ into itself. Since $A$ is the commutator algebra of $C_{m}$ in $A_{m}=A \otimes_{c} C_{m}$, it follows that $s-u$ must also map $A$ into itself, and therefore provides the desired extension of $t$ to a derivation of $A$.

Hence we may now assume that $A$ is a crossed product $Z(G, f)$, where $Z$ is a normal extension field of $C, G$ is the Galois group of $Z / C$, and $f$ is a factor set, i.e., a map of $G \times G$ into the multiplicative group of the nonzero elements of $Z$ such that, for all $\rho, \sigma, \tau$ in $G, \rho(f(\sigma, \tau)) f(\rho \sigma, \tau)^{-1} f(\rho, \sigma \tau) f(\rho, \sigma)^{-1}=1$. More explicitly, $Z(G, f)$, as a left vector space over $Z$, has a basis $u_{\sigma}$ in one to one correspondence with the elements $\sigma$ of $G$ such that $u_{\sigma} u_{\tau}=f(\sigma, \tau) u_{\sigma \tau}$, and, for $z \in Z, u_{\sigma} z=\sigma(z) u_{\sigma}$. Since $Z$ is separable over $C$, there is one and only one derivation of $Z$ which coincides with the given derivation $t$ of $C$. We shall denote this extended derivation of $Z$ by the same letter $t$. Clearly, the map $\sigma t \sigma^{-1}$ is also a derivation of $Z$ which extends our given derivation of $C$, whence $\sigma t \sigma^{-1}=t$. In other words, the extended derivation $t$ to $Z$ is also a $G$-endomorphism of $Z$. Now we define a map $h$ of $G \times G$ into $Z$ by setting $h(\sigma, \tau)$ $=t(f(\sigma, \tau)) f(\sigma, \tau)^{-1}$. Using that $t$ is a derivation and a $G$-endomorphism, we

(3) In the case where $C$ is finite over the subfield annihilated by the given derivation (and hence is of characteristic $\neq 0$ and purely inseparable of exponent 1 over that subfield), this theorem was already proved by Jacobson in [5]; one merely has to combine (in [5]) Theorem 7 with the part of $\$ 10$ that precedes Theorem 13, using the argument of the first paragraph of our proof below. The rest of our proof is quite different from that of Jacobson. 
derive from the above multiplicative identity for $f$ the corresponding additive identity for its logarithmic derivative, $h$, i.e., $\rho(h(\sigma, \tau))-h(\rho \sigma, \tau)+h(\rho, \sigma \tau)$ $-h(\rho, \sigma)=0$. It is a well-known result (due to Witt) that such an additive factor set must be a transformation set, i.e., that there is a map $g$ of $G$ into $Z$ such that $\sigma(g(\tau))-g(\sigma \tau)+g(\sigma)=h(\sigma, \tau)$. Indeed, there is an element $z_{1} \in Z$ whose trace is 1 , i.e., $\sum_{\sigma \in G} \sigma\left(z_{1}\right)=1$. Define $g(\rho)=\sum_{\sigma \in G} \rho \sigma\left(z_{1}\right) h(\rho, \sigma)$. Then it is easily verified that $g$ satisfies the above requirement. Now we define the additive endomorphism $t^{*}$ of $Z(G, f)$ by the formula

$$
t^{*}\left(\sum_{\sigma \in G} z_{\sigma} u_{\sigma}\right)=\sum_{\sigma \in G}\left(t\left(z_{\sigma}\right)+z_{\sigma} g(\sigma)\right) u_{\sigma}
$$

where the $z_{\sigma}$ are elements of $Z$. It is easy to check directly that $t^{*}$ is a derivation of $Z(G, f)$ which coincides with $t$ on $C$. This completes the proof of Theorem 6.

We shall now indicate briefly how Theorem 5 can be obtained from Theorem 6 and the theory of restricted Lie algebras. It is evidently sufficient to prove Theorem 5 in the case where $C^{p} C F$. In view of Theorem 3, it suffices then to show that there always exists a regular extension $(S, \phi)$ of $A$ by the derivation algebra $T$ of $C / F$, where $C$ is the center of $A$. As in the proof of Theorem 3 , we write $C=F\left[u_{1}, \cdots, u_{k}\right]$, and consider the derivations $t_{1}, \cdots, t_{k}$ of $C / F$ such that $t_{i}\left(u_{j}\right)=\delta_{i j} u_{j}$. If $T_{0}$ is the subspace of $T$ which is spanned, over $F$, by these elements $t_{i}$ then $T_{0}$ is a restricted abelian Lie algebra over $F$, the $p$-map in $T_{0}$ is nondegenerate in the sense that the image of $T_{0}$ under the $p$-map spans $T_{0}$ over $F$, and we have $T=C T_{0}$. The key result for our proof is that the restricted cohomology groups of $T_{0}$ are trivial. This was shown in $\$ 1$ of [4]. Before we can make use of this fact, we must modify the $p$-map in $A$. Let $a \rightarrow a^{\prime}$ be any $p$-semilinear of $A$ into $F$ such that $c^{\prime}=c^{p}$, for every $c \in C$. (The phrase $p$-semilinear means that, for $a, b$ in $F$ and $u, v$ in $A$, we have $(a u+b v)^{\prime}=a^{p} u^{\prime}+b^{p} v^{\prime}$.) We define a new $p$-map by setting $a^{[p]}=a^{p}-a^{\prime}$. With this $p$-map, and the usual commutation, $A$ is a restricted Lie algebra, which we shall denote by $A_{0}$. Our new $p$-map annihilates the center $C$ of $A_{0}$. By Theorem 6, every element of $T_{0}$ can be extended to a derivation of the associative algebra $A$, and such a derivation is then also a restricted derivation of the restricted Lie algebra $A_{0}$. Since the extension of a derivation of $C$ to a derivation of $A$ is unique up to an inner derivation of $A$, we thus have the structure of a restricted $T_{0}$-kernel on $A_{0}$, in the sense of [3]. Since the $p$-map in $A_{0}$ annihilates the center of $A_{0}$, and since the restricted 3-dimensional cohomology group for $T_{0}$ in $C$ is ( 0 ), it follows from Theorem 3.1 of [3] that the kernel $A_{0}$ is extendable, i.e., that there exists a restricted Lie algebra extension $\left(S_{0}, \phi_{0}\right)$ of $A_{0}$ by $T_{0}$ which induces our kernel structure on $A_{0}$, and in particular satisfies the regularity condition $\left({ }^{*}\right)$. Now we extend the map $a \rightarrow a^{\prime}$ to a $p$-semilinear map of $S_{0}$ into $F$ and then redefine the $p$-map in $S_{0}$ by $s \rightarrow s^{[p]}+s^{\prime}=s^{p}$, where $s \rightarrow s^{[p]}$ is the original $p$-map in $S_{0}$. There results a 
restricted extension $\left(S_{1}, \phi_{1}\right)$ of $A$ by $T_{0}$, where $A$ is now again regarded as a restricted Lie algebra in the usual way, $S_{1}$ is the same as $S_{0}$ except for the changed $p$-map, and $\phi_{1}$ is the same map as $\phi_{0}$. The regularity condition $\left(^{*}\right)$ continues to hold in $S_{1}$, of course. The same argument which gave the proof of Theorem 2 (which uses the vanishing of the 2-dimensional cohomology groups of $T_{0} ; \mathrm{cf}$. [4]) shows that there is an ordinary Lie algebra isomorphism $\psi$ of $T_{0}$ into $S_{1}$ which is inverse to $\phi_{1}$. Now we enlarge $S_{1}$ to a Lie algebra $S$ whose space is $A+C \otimes_{F} \psi\left(T_{0}\right)$, and where the commutation is that extension of the commutation in $S_{1}$ which results from enforcing the regularity condition (i), with the natural $C$-space structure of $S$. There remains to define a $p$-map in $S$. For $s_{i}=\psi\left(t_{i}\right)$ and $c \in C$, define $\left(c \otimes s_{i}\right)^{p}=c^{p} s_{i}^{p}+\left(c t_{i}\right)^{p-1}(c) \otimes s_{i}$. Then the inner derivation effected by $\left(c \otimes s_{i}\right)^{p}$ coincides with the $p$ th power of the inner derivation effected by $c \otimes s_{i}$. Indeed, this holds on $A$, by Lemma 1 of [4], and since $C \otimes_{F} \psi\left(T_{0}\right)$ is isomorphic with $T$, this holds also on $C \otimes_{F} \psi\left(T_{0}\right)$. Now it follows from the elementary theory of restricted Lie algebras that there is one and only one $p$-map on $S$ which coincides with the map $a \rightarrow a^{p}$ on $A$ and which maps the elements $c \otimes s_{i}$ into $\left(c \otimes s_{i}\right)^{p}$. Clearly, this $p$-map is an extension of the given $p$-map on $S_{1}$. Furthermore, the analysis of the regularity condition (ii) which we made immediately before stating Theorem 4 shows that $S$ satisfies the regularity condition (ii). Finally, $\phi_{1}$ can be extended in one and only one way to a $C$-linear map $\phi$ of $S$ onto $T$, and it is clear that $(S, \phi)$ is a regular extension of $A$ by $T$. This completes our proof for the existence of regular extensions.

\section{REFERENCES} 1952.

1. A. A. Albert, Structure of algebras, Amer. Math. Soc. Colloquium Publications, vol. 24,

2. G. Hochschild, Cohomology of restricted Lie algebras, Amer. J. Math. vol. 76 (1954) pp. 555-580.

3. - Lie algebra kernels and cohomology, Amer. J. Math. vol. 76 (1954) pp. 698-716.

4. - Simple algebras with purely inseparable splitting fields of exponent 1, Trans. Amer. Math. Soc. vol. 79 (1955) pp. 477-489.

5. N. Jacobson, Abstract derivation and Lie algebras, Trans. Amer. Math. Soc. vol. 42 (1937) pp. 206-224.

UNIVERSITY OF ILLINOIS

URBANA, ILL. 